\title{
Role of Trimetazidine in Coronary Artery Bypass Graft Surgery
}

\author{
Gerez Fernandes Martins ${ }^{1^{*}}$, Gerez Martins $^{2}$ \\ ${ }^{1}$ Cardiac Surgery Department, Institute of Cardiology State Aloysio de Castro, Rio de Janeiro, Brazil \\ ${ }^{2}$ Cardiology Department, Institute of Cardiology State Aloysio de Castro, Rio de Janeiro, Brazil \\ Email:*gerezm@cardiol.br
}

How to cite this paper: Martins, G.F. and Martins, G. (2017) Role of Trimetazidine in Coronary Artery Bypass Graft Surgery. World Journal of Cardiovascular Surgery, 7, 87-102.

https://doi.org/10.4236/wjcs.2017.77011

Received: May 31, 2017

Accepted: July 23, 2017

Published: July 26, 2017

Copyright (c) 2017 by authors and Scientific Research Publishing Inc. This work is licensed under the Creative Commons Attribution International License (CC BY-NC).

http://creativecommons.org/licenses/by/4.0/

(c) (i) Open Access

\begin{abstract}
Trimetazidine is a drug that has been used to reduce the damage of reperfusion injury, which is a mechanism of oxidative aggression that occurs in coronary syndromes, angioplasty procedures, as well as in cardiovascular surgeries. Seeking to improve myocardial protection during procedures that involve reperfusion, attention was recently turned to research on cytoprotective drugs which act on segments of cellular metabolism, used in isolation or combined to reduce or prevent damage to the cardiac cell. The aim of this review is to objectively evaluate the effects of the drug trimetazidine (TMZ) on ischemia-reperfusion injury in coronary artery bypass graft surgery by reviewing all known studies from recent years.
\end{abstract}

\section{Keywords}

Ischemia-Reperfusion Injury, Oxidative Stress, Inflammation, Trimetazidine, Coronary Artery Bypass Graft

\section{Introduction}

Prolonged ischemia, as with the evolution of an acute myocardial infarction, coronary bypass operation, or cardiac transplantation, jeopardizes cell viability and ultimately cardiac function [1] [2]. Myocardial ischemia of limited duration, less than 20 minutes, followed by reperfusion, is accompanied by functional recovery without evidence of structural or biochemical evidence of tissue injury. Paradoxically, reperfusion of cardiac tissue, which has been subjected to an extended period of ischemia ( $>20$ minutes), results in a phenomenon known as myocardial reperfusion injury [1] [2] [3].

Myocardial reperfusion injury is defined as the death of myocytes, alive at the time of reperfusion, as a direct result of one or more events initiated by reperfu- 
sion. Therefore, myocardial cell damage results from the restoration of blood flow to the previously ischemic heart thereby extending the region of irreversible injury beyond that due to the ischemic insult alone [1] [4] [5]. Reperfusion injury may also be defined as "those metabolic, functional, and structural consequences of restoring coronary arterial flow that can be avoided or reversed by modification of the conditions of reperfusion" [3].

The cellular damage in reperfusion injury can be reversible or irreversible, depending on the length of the ischemic insult. If reperfusion is initiated within 20 minutes after the onset of ischemia, the resulting myocardial injury is reversible and is characterized functionally by depressed myocardial contractility, which eventually recovers completely. Myocardial tissue necrosis is not detectable in the previously ischemic region although functional impairment of contractility may persist for a variable period, as myocardial stunning. Initiating reperfusion after a duration of ischemia of longer than 20 minutes, however, results in irreversible myocardial injury or cellular necrosis [1] [4] [5].

Irreversible reperfusion myocardial injury is defined as reperfusion-induced cell death of the cardiomyocytes that were still viable at the time of blood flow restoration [1]. Thus, irreversible reperfusion injury extends the initial infarction caused by ischemia with further clinical deterioration in the shape of heart failure and arrhythmias [1] [4] [5].

Reperfusion injury is a phenomenon which has been attracting the attention of researchers because of its evident and important repercussions in clinical cardiology practice, and has been leading to a better understanding of the events related to it, particularly as to the so-called hyperoxide radicals, that may cause injury after aorta unclamping in cardiac surgery. The hyperoxide radicals include superoxide anion $\left(\mathrm{O}_{2}^{-}\right)$, hydroxyl radical $(\mathrm{OH})$, and hydrogen peroxide $\left(\mathrm{H}_{2} \mathrm{O}_{2}\right)$, which can be removed from the cells by systems of enzymes with antioxidant functions, typically present in the myocardial physiology [4]. These systems of endogenous enzymes with antioxidant action, as superoxide dismutase, glutathione peroxidase, and catalase, are responsible for the limitation of intracellular accumulation of $\mathrm{O}_{2}^{-}$and $\mathrm{H}_{2} \mathrm{O}_{2}$ during normal metabolism, whether by inhibiting its formation or by carrying these radicals out of the cell, reducing the oxidative damage on proteins and lipids [4].

Seeking to improve myocardial protection during procedures that involve reperfusion injury, recent attention was turned to research on cytoprotective drugs which act on segments of cellular metabolism, used in isolation or combined to reduce or prevent damage to the cardiac cell. With its cytoprotective mechanism, TMZ limits calcium accumulation and intracellular acidosis, inflammation and hyperoxide free radicals production that occur after reperfusion, without provoking hemodynamic alterations. Its use in ventricular dysfunction prophylaxis has also demonstrated a beneficial action on the "stunned myocardium", a clinical translation of the injury over the ventricular function after myocardial revascularization surgery [5] [6] [7]. 
The purpose of this section is to evaluate the effects of the drug trimetazidine (TMZ) on the ischemia-reperfusion injury in the Cardiovascular Artery Bypass Grafting reviewing the studies from recent years [6].

\section{Mechanisms of Ischemia-Reperfusion Injury}

The major mechanisms of myocardial reperfusion injury are the absence of normal oxidative phosphorylation (Figure 1), oxidative stress (predominantly oxygen-free radicals), neutrophil-endothelium interactions, apoptosis, and calcium overloading [7]. It is unlikely that any single pathophysiologic mechanism of myocardial reperfusion injury causes lethal myocardial injury. When multiple pathologic events occur simultaneously, injury is an additive and the probability of irreversible myocardial injury occurring increases significantly [7].

Prolonged ischemia causes the absence of normal oxidative phosphorylation and diminished myocardial tissue concentrations of adenosine 5-triphosphate (ATP) and creatine phosphate within the mitochondria, which leads to ultrastructural changes [8] [9]. Initially, when ATP use exceeds the capacity of the myocytes to resynthesize high-energy phosphates, degradation of adenine nucleotides results in the conversion of adenosine diphosphate (ADP) to adenosine monophosphate (AMP) [8]. Further sustained reductions in ATP availability result in irreversible myocyte injury associated with sarcolemmal damage, acidosis, and cellular swelling [8]. Myocardial ischemia is further associated with an accumulation of intracellular lactic acid [10] and a decline in fatty-acid oxidation, which normally provides $60 \%$ to $90 \%$ of myocardial energy requirements [11].

\subsection{Oxidative Stress}

The oxygen paradox hypothesis is based upon the basis that oxygen can inflict

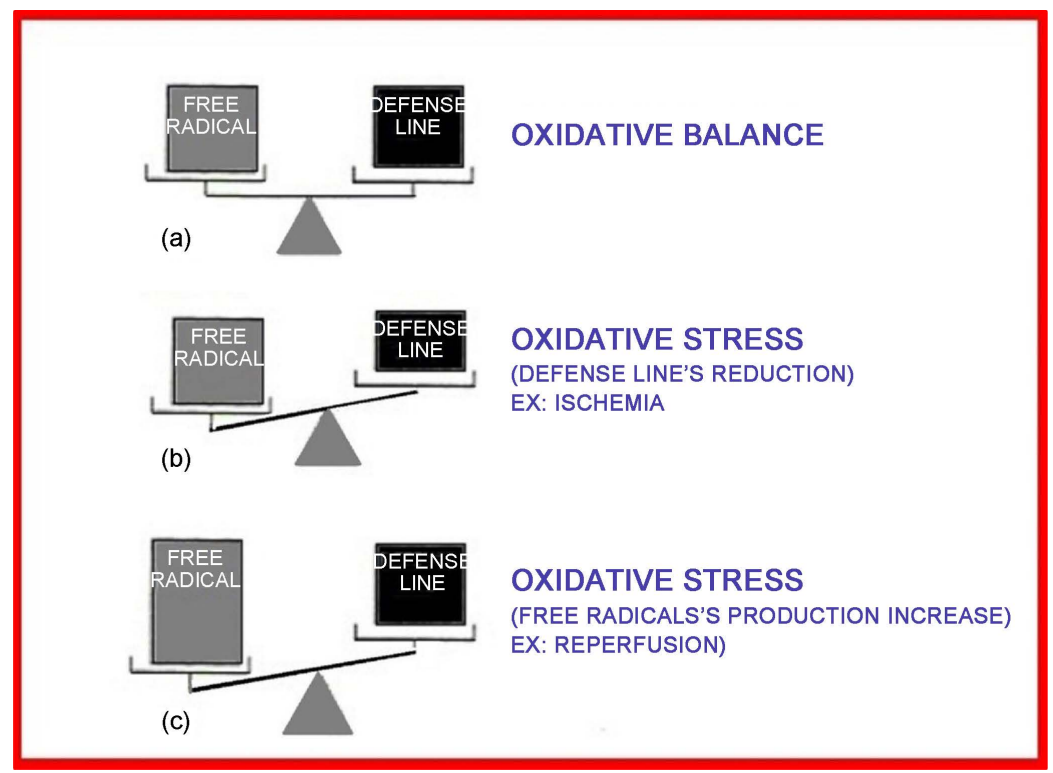

Figure 1. Oxidative balance Brasileiro (1997, p. 84). 
injury to the ischemic myocardium during reperfusion [5]. Upon reperfusion, myocytes go from a hypoxic state to normal oxygen tension very rapidly, thus initiating the sequential reduction of $\mathrm{O}_{2}$ resulting in reactive oxygen species production [5] [12]. Cells that encounter the reactive oxygen species (ROS) undergo oxidative damage as the ROS becomes more stable at the expense of nearby molecules in contact. Under physiologic conditions, small quantities of ROS are formed during mitochondrial respiration, but they can be detoxified by endogenous scavenging mechanisms [13]. After reperfusion many scavenging molecules are "washed out" of the areas in which ROS protection is critically needed [14] [15]. Normally, hypoxanthine produced from AMP metabolism is further metabolized by xanthine dehydrogenase (XD) to xanthine [16]. During ischemia, however, $\mathrm{XD}$ is converted to xanthine oxidase (XO) by calcium $\left(\mathrm{Ca}^{2+}\right)$ dependent proteases (Figure 2).

ROS can cause activated neutrophils, variations within the mitochondrial electron chain, reduced contractility, autoxidation of catecholamines, the activation of the arachidonic cascade, cyclooxygenase, and lipoxygenase, lipid peroxidation, Deoxyribonucleic acid (DNA) damage and impairment of mitochondrial function, which can progress to myocyte structural damage [5] [14].

\subsection{Calcium Overload Theory}

The initiation and upshot of the action potential in myocardium is derived from the entry of sodium ions $\left(\mathrm{Na}^{+}\right)$across the sarcolemma in a positive feedback loop. The influx of extracellular $\mathrm{Ca}^{2+}$ ions through L-type calcium channels (LTCC) (also known as dihydropyridine receptors, DHP) sustains the depolarization of cardiomyocytes for a longer duration [17] [18]. Calcium-induced calcium release from the sarcoplasmic reticulum (SR) occurs under normal excitation-contraction (EC) coupling. After increases of intracellular $\mathrm{Ca}^{2+}$ ions concentrations, $\mathrm{Ca}^{2+}$ ions bind to the protein troponin, which initiates contraction by allowing the contractile proteins, myosin and actin, to associate through cross-bridge formation. Their outflow into SR is regulated via the cardiac ryanodine receptor type $2(\mathrm{RyR} 2)$ and this function is dependent on the

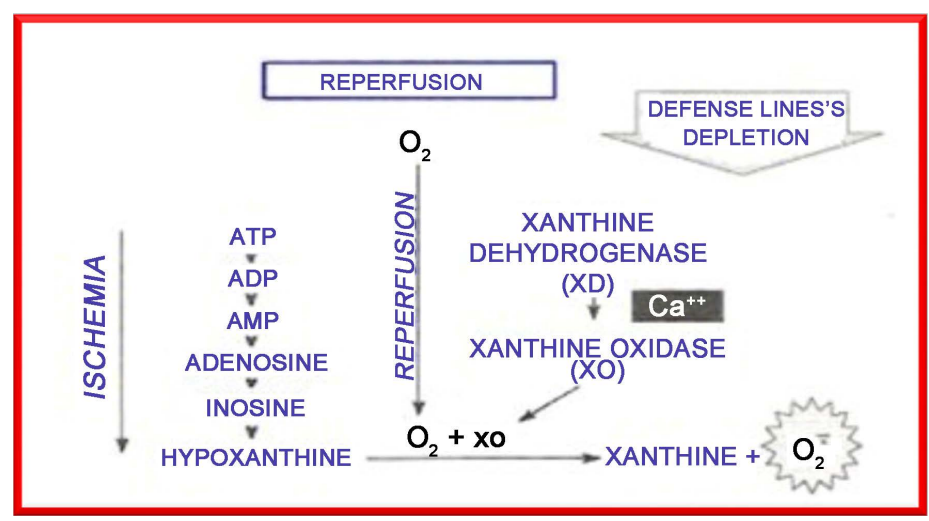

Figure 2. Reperfusion mechanism. Brasileiro (1997, p. 86). 
cytosolic-located stabilizing proteins FKBP12 and FKBP12.6 and their connection to RyR2 [17] [18].

In heart failure and ischemic-reperfusion injury, this regulation of the $\mathrm{Ca}^{2+}$ flow malfunctions and FKBP12 has been shown to dissociate from the RyR2. This allows the outflow of $\mathrm{Ca}^{2+}$ ions to increase out from the SR into the cytosol. Increased cytosolic calcium can activate protein kinases, phospholipases and other degradative enzymes [17] [19] [20].

\subsection{The Neutrophil-Endothelial Interactions}

During myocardial ischemia-reperfusion, neutrophils are activated by inflammatory molecules released by cardiac myocytes, endothelial cells, and mast cells, resulting in a neutrophil attack against itself [21] [22]. Neutrophils are activated by complement C5a and cytokines such as interleukins (IL), IL-6, IL-8, tissue necrosis factor-alpha (TNF- $\alpha$ ), neutrophil-activating peptide, and platelet activating factor [7].

Neutrophils contribute to myocardial reperfusion injury by the production of ROS, proteolytic enzymes and cytokines, endothelial dysfunction, capillary plugging, and direct myocyte injury [21] [23]. Neutrophils also cause coronary vascular constriction, which can decrease cardiac performance [24]. Through a process called "no-reflow", aggregating neutrophils can occlude microvessels and increase capillary blood flow resistance, causing microcirculatory ischemia and even tissue infarction [25]. No-reflow is defined as severe microvascular dysfunction that limits perfusion during reperfusion [26].

Endothelial dysfunction occurs in the first 2.5 to 5 minutes of reperfusion. The effects of endothelial dysfunction include platelet and neutrophil activation, vasoconstriction, increased ROS production, and increased protein and fluid extravasation [26].

\subsection{Mitochondrial Dysfunction and Apoptosis}

Mitochondrial permeability transition pore (MPTP), mitochondrial dysfunction and apoptosis.

Normally, the respiratory chain releases only small amounts of ROS pre-dominantly by complex I. During early reperfusion, ROS formation from various sources, including the respiratory chain, is largely augmented. Mitochondrial complexes I and III are major sources of pathological ROS production. The MPTP is a large-conductance mega-channel putatively constituted by the voltage-dependent anion channel in the outer membrane, the adenine nucleotide transporter in the inner membrane, and cyclophilin D in the matrix. Under physiological conditions, MPTP is predominantly in a closed state [27]. The opening of the MPTPs causes the disruption of the inner mitochondrial membrane. The membrane will lose its potential, a depletion of the mitochondrial nicotinamide adenine dinucleotide (NADH) pool will occur, and the oxidative phosphorylation will be uncoupled leading to accelerating a source of ROS, and can lead to 
further cascade reactions with fatal cell injuries as a consequence. The early opening of the MPTPs also activates the mitochondrial apoptosis cascade which is yet another mechanism for accelerated cell death following ischemia and reperfusion [28].

\subsection{Trimetazidine}

Trimetazidine, [1-(2,3,4-trimethoxybenzyl)piperazine dihydrochloride](TMZ) (Figure 3), is a metabolic anti-ischemic drug that exerts its beneficial effects without altering the hemodynamic function of the heart [29]. The mechanism of action of trimetazidine is related to the optimising cardiac metabolism by reducing fatty acid oxidation through the selective inhibition of mitochondrial 3-ketoacyl CoA thiolase, which is critical to the $b$-oxidation pathway. As a result, TMZ decreases ischemic stress and improves cardiac performance during ischemia [30]. This inhibition decreases the utilisation of free fatty acids as a source of energy for the myocardium, resulting in an increase in glucose oxidation (Figure 4). This metabolic switch acts to improve cardiac energy metabolism by switching ATP production from lipid to glucose oxidation, thus enhancing intramitochondrial coupling and favouring a more efficient mode of ATP production per mole of oxygen [29] [30]. At the cellular level, TMZ preserves ATP production and reduces intracellular acidosis and calcium overload and thereby maintains the cellular homeostasis [30]. TMZ decreases oxidative damage to mitochondria and protects hearts from I/R-induced damage to mitochondrial respiration [31]. TMZ also showed a cytoprotective effect in several models of myocardial infarction [32] [33]. Recently, it has been shown that TMZ protected post-ischemic hearts by inhibiting the activation of neutrophils [34].

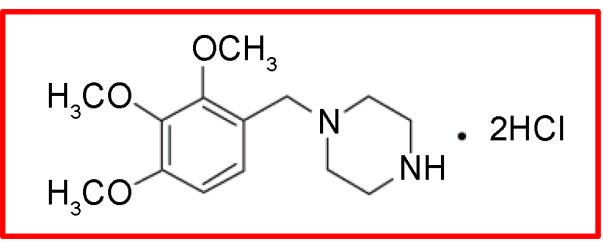

Figure 3. Chemical structure of trimetazidine dihydrochloride. J Bioequiv Availab (2011).

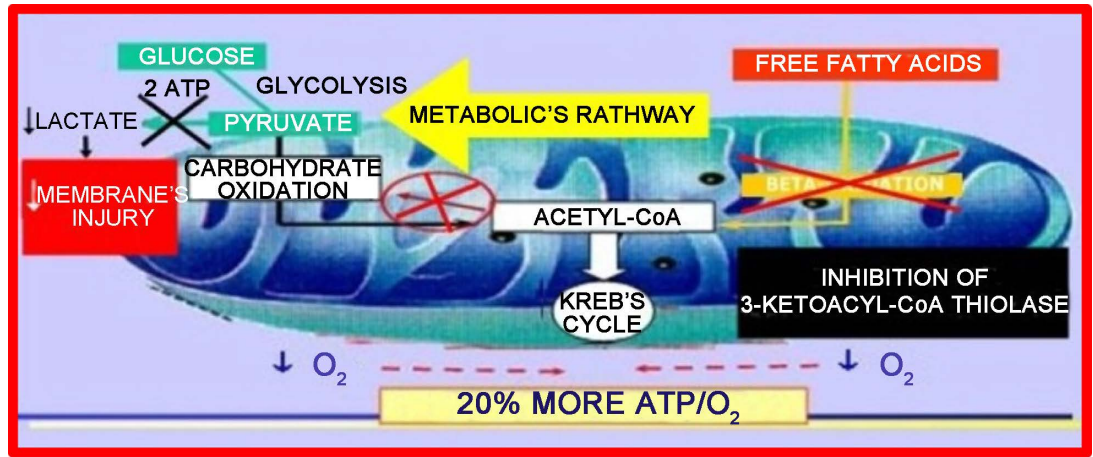

Figure 4. The mechanism of action of trimetazidine, Banani et al. (1998). 


\subsection{TMZ in Coronary Artery Bypass Grafting Cardiosurgery}

There are still only a few studies suggesting the role of TMZ in patients subjected to coronary artery bypass grafting (CABG) in the last 10 years. However, according to the studies with reperfusion protection effects of TMZ in patients undergoing percutaneous transluminal coronary angioplasty (PTCA) and anti-ischemic role during stable angina confirmed in guidelines, the application of TMZ in patients undergoing surgical revascularization seems to be completely justified [35].

Nowadays the chronic coronary disease has good evidence in their treatment, supported by guidelines and meta-analysis of randomised double-blind trials. The group of patients treated with PTCA after aggressive medical therapy and TMZ, showed less ST elevation and significant lower mean $(P<0.05)$ and maximal amplitude of $\mathrm{T}$ wave alteration. The trimetazidine administered before angioplasty is sustained by few randomised studies with good myocardial protection [36].

Finally, in the first randomised, double-blind, placebo-controlled study, developed in 1992, Fabiani et al. [37] evaluated the role of TMZ in the prevention of disadvantageous effects of ischemia-reperfusion injury in patients subjected to heart surgery. Nineteen patients (10 in TMZ group, and nine in the control group) who underwent CABG were included in the study. TMZ was started at $20 \mathrm{mg}$, three times daily orally for three weeks before the surgery and the same drug was added to the cardioplegic solutions [37]. Metabolic measurements showed that the increase of malondialdehyde in the coronary sinus 20 minutes after reperfusion was significantly reduced in the trimetazidine group ( $p=$ 0.014). Myosin was present later in the TMZ group compared to the placebo group $(p=0.036)$. The obtained outcomes showed that patients pretreated with TMZ had better ventricular function, as assessed by the work stroke index (SWI), which was significantly higher in the TMZ group than in the placebo group $(p=0.01)$. Initially with the first pretreatment with TMZ it seemed to reduce ischemia-reperfusion injury and developed improved ventricular function after surgery [37]. Similar results were obtained in the experimental study by Aussedat et al. (1993) [38] and later with Banani et al. (2000) [39], confirmed its role as an addiction to the cardioplegic solution; it reduced the effects of ischemia-reperfusion injury [38] [39].

The second randomised, double-blind, placebo-controlled study by Vendrinne et al. (1996) [40], obtained results which were definitely less effective with the pretreatment of TMZ on CABG. The authors assessed the cardioprotective effects of TMZ on left ventricular function after surgery. Forty patients undergoing elective $\mathrm{CABG}$, receiving either TMZ or a placebo $(\mathrm{PCB})$, were included. The serial measurements of fractional area change (FAC), percent of systolic wall thickening (SWT) and malondialdehyde (MDA) were performed in all patients. There were no differences regarding the number of vessels revascularized, the duration of aortic clamping or bypass time. The authors showed no FAC 
differences between the two groups. SWT was lower in the TMZ group.MDA increased by $24 \%$ in the PCB group and $25 \%$ in the TMZ group. Lactate levels were lower in the TMZ group $(p<0.05)$. The authors concluded that in patients with good pre-operative ejection fraction, TMZ did not demonstrate clinically significant cardioprotective effects on left ventricular performance and lipid peroxidation compared to placebo [40]. However, this study had many limitations, so the results should be confirmed in future studies, as pointed out in comments after publication. Later we will review the protective properties of TMZ in patients with normal or mildly dysfunctional ventricle in the study of Martins et al. $2011[41]$.

Three years later Turnerir et al. (1999) [42] observed the cardioprotective role of TMZ. The authors evaluated potential myocardial protection of TMZ by measurement of troponin $t(\mathrm{TnT})$ during CABG. Thirty randomised patients were included in this double-blind study. The TMZ group was composed of 15 patients in New York Heart Association (NYHA) class III or IV. Pretreatment was started three weeks before CABG with TMZ (60 mg orally per day) or placebo. The pre-operative serum concentration of TnT levels were 0 to $0.39 \mathrm{ng} / \mathrm{mL}$ in all patients. The mean TnT levels were measured five mins after completion of cardiopulmonary bypass and 12, 24 and $48 \mathrm{~h}$ post-operatively in the placebo and TMZ groups. The result shows that in the TMZ group, TnT levels were significantly lower than those in the placebo group at all times $(p=0.001)$. The authors concluded that pretreatment with TMZ significantly reduces ischemic-reperfusion damage during CABG [42].

Nevertheless we should attempt to quantify these results, evaluated in clinical improvement of the patients and performing the action which allows analysis of the outcomes and its relation to the pretreatment conduced with trimetazidine.

Kuralay et al. (1999) [43] evaluated the effect of trimetazidine and diltiazem on persistent myocardial ischemia after coronary artery bypass graft surgery in the prospective randomised study. Sixty patients were divided into three groups of 20 each and followed up for 12 months. Patients in all three groups received acetylsalicylic acid $100 \mathrm{mg}$ per day, those in group one also had trimetazidine 60 mg per day, and those in group two had diltiazem $90 \mathrm{mg}$ per day. Each patient had coronary angiography during the first three weeks after surgery, 24-hour ambulatory monitoring every month, and cardiac scintigraphy at three weeks, six months, and 12 months. Perfusion defects had resolved in the trimetazidine group at six months. At 12 months, perfusion defects had resolved in the diltiazem group but not in the group receiving only acetylsalicylic acid $(p<0.05)$. Based on these results it seems that trimetazidine is superior to diltiazem in resolving the myocardial perfusion defects at six months after coronary artery bypass surgery [43].

Ruzyllo et al. (2004) showed by retrospective analysis of a subgroup of 94 patients from the TRIMPOL II study that trimetazidine provides anti-anginal efficacy in post-revascularized patients with recurrent angina despite a monotherapy with metoprolol [44]. 
More recently, Banach et al. (2005) [45] evaluated the effect of TMZ on the haemodynamic parameters of the heart and post-operative prognosis. One hundred and forty patients were included in the study, divided into two groups (placebo and TMZ), receiving medication/placebo at a dose of $20 \mathrm{mg}$ three times a day. The therapy was used for at least two months before and after cardiac surgery. The following variables were evaluated during the study: mean time of aortic clamping, post-operative levels of creatine kinase isoenzyme $\mathrm{MB}$ (CK-MB), early post-operative ejection fraction (EF), post-operative arrhythmias occurrence, mean time of intensive care unit (ICU) stay and length of hospitalisation. The authors did not observe significant differences in the number of post-operative arrhythmias, but atrial fibrillation was less frequent in the TMZ group compared to the placebo group $(p=0.05)$. The pre-operative ejection fraction was similar in both groups; however, in the post-operative period, a significant increase of EF in TMZ group was found $(p=0.05)$. Differences in early mortality in both groups were not observed. The authors conclude that administration of TMZ before and after cardiac surgery might influence post-operative haemodynamic parameters [45].

Iskensen et al. (2006) [46] investigated the effect of pre-operative use of TMZ on the reduction of oxidative stress during CABG. Twenty-four patients were included in the study, divided into two groups (control and TMZ). The control group did not receive any medication and the TMZ group received pretreatment two weeks before CABG (60 mg per day). Serial blood samples were collected before and after CABG for measurement of the serum concentrations of major endogenous antioxidant enzyme systems. Hemodynamic parameters were measured before operation, just after $\mathrm{CPB}$, and at one and 16 hours after $\mathrm{CPB}$ using thermodilution technique and radial arterial and central venous pressure. Serial venous blood samples were collected two weeks pre-operatively, just before operation, and at one min and 15 mins after release cross-clamp. Serum Superoxide dismutase (SOD), glutadione peroxidase (GPx) endogenous antioxidant enzyme systems and the end product of lipid peroxidation, malondialdehyde (MDA), were measured as oxidative stress markers. The authors showed that post-operative levels of antioxidant enzymes were significantly different between the groups $(p=0.05)$, higher in the TMZ group, but MDA levels were significantly higher in the control group. There were no significant differences between the two groups in the hemodynamic parameters. The authors concluded that pretreatment with TMZ preserves endogenous antioxidant capacity during CABG [46].

Martins et al. (2008) [41] evaluated the effect of TMZ on ischemic-reperfusion injury. The aim of this study was to determine whether trimetazidine could reduce myocardial reperfusion injury damage in coronary artery bypass graft surgery (CABG) using evolutive serial troponin $\mathrm{T}$ and creatine kinase isoenzyme $\mathrm{MB}$ (CPK-MB) blood levels and to verify possible follow-up changes in left ventricular (LV) echocardiographic function, and also the frequency of the ventri- 
cular fibrillation at the end of bypass. The authors conduced a double-blind, placebo-controlled study on 60 randomised patients who underwent CABG. The TMZ and the placebo group were composed of 30 patients each, stratified by echocardiography to include only patients with normal and/or mild LV dysfunction and receiving medication/placebo at a dose of $20 \mathrm{mg}$ three times a day, starting from 12 to 15 days after a pre-operative period of up to five to eight days after the post-operative period. Troponin $\mathrm{T}$ and CPK-MB were measured pre-operatively without medication, 12 to 15 days of medication/placebo taken five minutes after aortic declamping, and at subsequent 12, 24 and 48 hours. The echocardiographic measurements were performed: pre-operative without medication, on the day of surgery with the use of 10 days of medication/placebo, and after leaving the ICU with at least 15 days of medication/placebo. The ventricular fibrillation was verified through cardioscopic and direct transthoracic observation at the end of bypass. The results of TnT and CPK-MB reached highly significant values $(p=0.0001)$ in the treated group compared to the control group at the four points when analysed-five mins, $12 \mathrm{~h}, 24 \mathrm{~h}$ and $48 \mathrm{~h}$. The echocardiographic variables did not show evolutive changes in each group which were considered and compared between themselves. The frequency of ventricular fibrillation at the end of bypass was low in both groups. The authors conclude that pretreatment with trimetazidine reduces ischemic-reperfusion damage during CABG, but did not affect LV echocardiographic variables [41].

In the latter trial to present, Lopatin and Dronova (2009) [47] could add the greatest number of patients in these last 10 years of study, as well as the longer period of follow-up and using a new presentation of trimetazidine $(35 \mathrm{mg} / \mathrm{MR}$ per tablet) evaluating clinical and pharmacoeconomic effects of long-term use of trimetazidine $\mathrm{MR}$ in 306 patients undergoing coronary artery bypass grafting (CABG). Six hours after CABG serum creatine Kinase and creatine-Kinase MB were significantly lower in the group using trimetazidine during two weeks prior to surgery. The rate of ischemic events was also lower in this patients and the long-term use of trimetazidine MR improve left ventricular systolic function and exercise tolerance [47].

Recently, in a randomised double-blind prospective study, controlled with placebo, the effects of trimetazidine (TMZ) on the inflammatory response were analysed. The changes in inflammatory response were analysed by using the variation in the levels of leukocytes, neutrophils and monocytes, and also in the interleukins 6 and 8, TNF- $\alpha$, complements $\mathrm{C} 3$ and C5, and highly sensitive C-reactive protein (HS-CRP) levels in the pre- and post-operative periods. In this study 30 patients were randomly selected, with no more than a mild ventricular dysfunction, and divided into two groups (TMZ and placebo) stratified by echocardiography and receiving medication/placebo in a $60 \mathrm{mg} /$ day dose. Measurements were taken on the pre-operative day without medication, on surgery day with 12 to 15 days of medication/placebo, within five minutes after the aortic declamping, and within 12, 24 and 48 hours after surgery. The results of this study showed that leukocytes and neutrophils levels were significantly decreased 
in the treated group when compared to the control group $(p=0.012 ; p=0.005)$. No significant differences in the levels of interleukin 8, TNF- $\alpha, \mathrm{C} 3$ and C5, and HS-CRP between these groups were observed. However, the interleukin 6 levels were significantly lower in the group treated as compared with those in the control group at all time points assessed [48] [49].

Finally, Hei L et al. (2015), published a systematic review and meta-analysis of randomized controlled trials (RCTs) to investigate the effectiveness of myocardial preservation of preoperative trimetazidine therapy in CABG patients by assessing the postoperative levels of several blood-based biochemical markers of myovardial injury, including creatine kinase (CK), creatine kinase-muscle and brain (CK-MB), creatine phosphokinase (CPK), troponin $\mathrm{T}(\mathrm{Tn} T)$ and troponin I (TnI). Six RTCs were finally included in the meta-analysis and the results showed significantly lower postoperative levels of CK, CK-MB, TNT, and TnI in trimetazidine-treated group. In sum, preoperative trimetazidine therapy appears to have a positive effect on myocardial preservation in CABG patients [50].

\section{Conclusion}

There are still only a few studies suggesting the role of TMZ in patients subjected to CABG. However, we can verify that the meta-analyzes prove the action of the drug in the protection of the cardiac cell with improvement of the symptoms instable angina due to its anti-ischemic effect [51] [52] [53]; Furthermore, clinical trials with patients who developed heart failure and were treated with TMZ demonstrate an evident improvement in left ventricular function, with a consequent increase in exercise tolerance. With a well known mechanism of activity, the application of TMZ in patients undergoing surgical revascularization seems to be completely justified. Moreover, a systematic review and meta-analysis of randomized controlled trials confirm this thesis and previous research. These studies should not only present numerical results of pharmacological effects, but also represent improvements in clinical variables and their correlation with a positive effect on myocardial preservation during and after CABG treatment.

\section{Disclosure}

No conflict of interest.

\section{References}

[1] Yellon, D.M. and Hausenloy, D.J. (2007) Myocardial Reperfusion Injury. The New England Journal of Medicine, 375, 1121-1135. https://doi.org/10.1056/NEJMra071667

[2] Braunwald, E. and Kloner, R.A. (1985) Myocardial Reperfusion: A Double-Edged Sword. The Journal of Clinical Investigation, 76, 1713-1719. https://doi.org/10.1172/JCI112160

[3] Rosenkranz, E.R. and Buckberg, G.D. (1983) Myocardial Protection during Surgical Coronary Reperfusion. Journal of the American College of Cardiology, 1, 12351246. 
[4] Bolli, R. (1998) Basic and Clinical Aspects of Myocardial Stunning. Progress in Cardiovascular Diseases, 40, 477-516. https://doi.org/10.1016/S0033-0620(98)80001-7

[5] Park, J.L. and Lucchesi, B.R. (1999) Mechanisms of Myocardial Reperfusion Injury. The Annals of Thoracic Surgery, 68, 1905-1912. https://doi.org/10.1016/S0003-4975(99)01073-5

[6] Argaud, L., Gomes, L., Roesch, O., et al. (2005) Trimetazidine Inhibits Mitochondrial Permeability Transition Pore Opening and Prevents Lethal Ischemia-Reperfusion Injury. Journal of Molecular and Cellular Cardiology, 39, 893-899. https://doi.org/10.1016/j.yjmcc.2005.09.012

[7] Hoffman, J.W., Gilbert, T.B. and Poston, R.S. (2004) Myocardial Reperfusion Injury: Etiology, Mechanisms, and Therapies. Journal of the ExtraCorporeal Technology, 36, 391-411.

[8] Shernan, S.K. (2003) Perioperative Myocardial Ischemia Reperfusion Injury. Anesthesiology Clinics of North America, 21, 465-485. https://doi.org/10.1016/S0889-8537(03)00038-5

[9] Schaper, W. and Schaper, J. (1997) Reperfusion Injury. The Journal of Thrombosis and Thrombolysis, 4, 113-116. https://doi.org/10.1023/A:1017543727429

[10] Ganz, P., Abben, R. and Barry, W. (1987) Dynamic Variations in Resistance of Coronary Arterial Narrowings in Angina Pectoris at Rest. American Journal of Cardiology, 59, 66-70. https://doi.org/10.1016/S0002-9149(87)80071-1

[11] Camici, P., Marraccini, P. and Lorenzoni, R. (1991) Metabolic Markers of StressInduced Myocardial Ischemia. Circulation, 83, 8-13.

[12] Barandier, C., Tanguy, S., Pucheu, S., Boucher, F. and De Leiris, J. (1999) Effect of Antioxidant Trace Elements on the Response of Cardiac Tissue to Oxidative Stress. Annals of the New York Academy of Sciences, 874,138-155. https://doi.org/10.1111/j.1749-6632.1999.tb09232.x

[13] Ide, T., Tsutsui, H., Kinugawa, S., et al. (1999) Mitochondrial Electron Transport Complex I Is a Potential Source of Oxygen Free Radicals in the Failing Myocardium. Circulation Research, 85, 357-363. https://doi.org/10.1161/01.RES.85.4.357

[14] Ma, X., Gao, F., Lopez, B., Christopher, T. and Vinten-Johansen, J. (2000) Peroxynitrate, a Two-Edged Sword in Post-Ischemic Myocardial Injurydichotomy of Action in Crystalloid- versus Blood-Perfused Hearts. Journal of Pharmacology and Experimental Therapeutics, 292, 912-920.

[15] Das, D.K. and Maulik, N. (1994) Antioxidant Effectiveness in Ischemia-Reperfusion Tissue Injury. Methods in Enzymology, 233, 601-610. https://doi.org/10.1016/S0076-6879(94)33063-8

[16] Carden, D. and Granger, D. (2000) Pathophysiology of Ischemia-Reperfusion Injury. Journal of Pathology, 190, 255-266. https://doi.org/10.1002/(SICI)1096-9896(200002)190:3<255::AID-PATH526>3.0.CO;2-6

[17] Olson, E. (2004) A Decade of Discoveries in Cardiac Biology. Nature Medicine, 10, 467-474. https://doi.org/10.1038/nm0504-467

[18] Wehrens, X.H.T., Lehnart, S.E. and Marks, A.R. (2005) Intracellular Calcium Release and Cardiac Disease. Annual Review of Physiology, 67, 69-98. https://doi.org/10.1146/annurev.physiol.67.040403.114521

[19] Lehnart, S.E., Maier, L.S. and Hasenfuss, G. (2009) Abnormalities of Calcium Metabolism and Myocardial Contractility Depression in the Failing Heart. Heart Failure Reviews, 14, 213-224. https://doi.org/10.1007/s10741-009-9146-X

[20] Yano, M., Yamamoto, T., Ikemoto, N. and Matsuzaki, M. (2005) Abnormal Ryano- 
dine Receptor Function in Heart Failure. Pharmacology \& Therapeutics, 107, 377391. https://doi.org/10.1016/j.pharmthera.2005.04.003

[21] Vinten-Johansen, J. (2003) Involvement of Neutrophils in the Pathogenesis of Lethal Myocardial Reperfusion Injury. Cardiovascular Research, 61, 481-497. https://doi.org/10.1016/j.cardiores.2003.10.011

[22] Engler, R.L., Dahlgren, M.D., Peterson, M.A., Dobbs, A. and Schmid-Schönbein, G.W. (1986) Accumulation of Polymorphonuclear Leucocytes during 3h Experimental Myocardial Ischemia. American Journal of Physiology, 251, H93-H100.

[23] Jordan, J.E., Zhao, Z. and Vinten-Johansen, J. (1999) The Role of Neutrophils in Myocardial Ischemia-Reperfusion Injury. Cardiovascular Research, 43, 860-878. https://doi.org/10.1016/S0008-6363(99)00187-X

[24] Szekely, A., Heindl, B., Zahler, S., Conzen, P. and Becker, B. (2000) Nonuniform Behavior of Intravenous Anesthetics on Postischemic Adhesion of Neutrophils in the Guinea Pig Heart. Anesthesia \& Analgesia, 90, 1293-1300. https://doi.org/10.1097/00000539-200006000-00007

[25] Budde, J.M., Morris, C.D., Velez, D.A., et al. (2004) Reduction of Infarct Size and Preservation of Endothelial Function by Multidose Intravenous Adenosine during Extended Perfusion. Journal of Surgical Research, 116, 104-115. https://doi.org/10.1016/S0022-4804(03)00356-1

[26] Verma, S., Fedak, P.W., Weisel, R.D., et al. (2002) Fundamentals of Reperfusion Injury for the Clinical Cardiologist. Circulation, 105, 2332-2336. https://doi.org/10.1161/01.CIR.0000016602.96363.36

[27] Kruzliak, P., Pechanova, O. and Kara, T. (2013) New Perspectives of Nitric Oxide Donors in Cardiac Arrest and Cardiopulmonary Resuscitation Treatment. Heart Failure Reviews, 19, 383. https://doi.org/10.1007/s10741-013-9397-4

[28] Armstrong, J.S. (2006) The Role of the Mitochondrial Permeability Transition in Cell Death. Mitochondrion, 6, 225-234. https://doi.org/10.1016/j.mito.2006.07.006

[29] Lopaschuk, G.D., Barr, R., Thomas, P.D. and Dyck, J.R. (2003) Beneficial Effects of Trimetazidine in Ex Vivo Working Ischemic Hearts Are Due to a Stimulation of Glucose Oxidation Secondary to Inhibition of Long-Chain 3-Ketoacyl Coenzyme a Thiolase. Circulation Research, 93, e33-e37. https://doi.org/10.1161/01.res.0000086964.07404.a5

[30] Kantor, P.F., Lucien, A., Kozak, R. and Lopaschuk, G.D. (2000) The Antianginal Drug Trimetazidine Shifts Cardiac Energy Metabolism from Fatty Acid Oxidation to Glucose Oxidation by Inhibiting Mitochondrial Long-Chain 3-Ketoacyl Coenzyme a Thiolase. Circulation Research, 86, 580-588. https://doi.org/10.1161/01.RES.86.5.580

[31] Guarnieri, C. and Muscari, C. (1993) Effect of Trimetazidine on Mitochondrial Function and Oxidative Damage during Reperfusion of Ischemic Hypertrophied Rat Myocardium. Pharmacology, 46, 324-331. https://doi.org/10.1159/000139070

[32] Harpery, C., Clauser, P., Labrid, C., Freyria, J.L. and Poirier, J.P. (1989) Trimetazidine, a Cellular Anti-Ischemic Agent. Cardiovascular Therapeutics, 6, 292-312.

[33] Pantos, C., Bescond-Jacquet, A., Tzeis, S., et al. (2005) Trimetazidine Protects Isolated Rat Hearts against Ischemia-Reperfusion Injury in an Experimental Timing-Dependent Manner. Basic Research in Cardiology, 100, 154-160. https://doi.org/10.1007/s00395-004-0505-4

[34] Tritto, I., Wang, P., Kuppusamy, P., Giraldez, R., Zweier, J.L. and Ambrosio, G. (2005) The Anti-Anginal Drug Trimetazidine Reduces Neutrophil-Mediated Car- 
diac Reperfusion Injury. Journal of Cardiovascular Pharmacology, 46, 89-98. https://doi.org/10.1097/01.fjc.0000164091.81198.a3

[35] Danchin, N. (2006) Clinical Benefits of a Metabolic Approach with Trimetazidine in Revascularized Patients with Angina. American Journal of Cardiology, 98, 8-13. https://doi.org/10.1016/j.amjcard.2006.07.003

[36] Chen, Y.D., Zhao, L.K., Tian, F., et al. (2010) Evaluation of the Myocardial Protection of Trimetazidine during Percutaneous Coronary Intervention: A Multi-Centrerandomised and Controlled Clinical Study. Chinese Journal of Internal Medicine, 49, 473-476.

[37] Fabiani, J.N., Ponzio, O., Emerit, I., et al. (1992) Cardioprotective Effect of Trimetazidine during Coronary Artery Graft Surgery. Journal of Cardiovascular Surgery, 33, 486-491.

[38] Aussedat, J., Ray, A., Kay, L., et al. (1993) Improvement of Long-Term Preservation of Isolated Arrested Rat Heart: Beneficial Effect of the Anti-Ischemic Agent Trimetazidine. Journal of Cardiovascular Pharmacology, 21, 128-135. https://doi.org/10.1097/00005344-199301000-00019

[39] El-Banani, H., Bernard, M., Baetz, D., et al. (2000) Changes in Intracellular Sodium and $\mathrm{pH}$ during Ischemia-Reperfusion Are Attenuated by Trimetazidine. Comparison between Low- and Zero-Flow Ischemia. Cardiovascular Research, 47, 688-696. https://doi.org/10.1016/S0008-6363(00)00136-X

[40] Vendrinne, J.M., Vedrinne, C., Bompard, D., et al. (1996) Myocardial Protection during Coronary Artery Bypass Graft Surgery: A Randomised, Double-Blind, Placebo-Controlled Study with Trimetazidine. Anesthesia \& Analgesia, 82, 712-718.

[41] Martins, G.F., Siqueira-Filho, A.G., Santos, J.B.F., et al. (2011) Trimetazidine on Ischemic Injury and Reperfusion in Coronary Artery Bypass Grafting. Arquivos Brasileiros de Cardiologia, 97, 209-216. https://doi.org/10.1590/S0066-782X2011005000079

[42] Turnerir, B., Colak, O., Alatas, O., et al. (1999) Mesurement of Troponin T to Detect Cardioprotective Effect of Trimetazidine during Coronary Artery Bypass Grafting. Annals of Thoracic Surgery, 68, 2173-2176. https://doi.org/10.1016/S0003-4975(99)01126-1

[43] Kuralay, E., Demirkiliç, U., Özal, E., Uzun, M. and Tatar, H. (1999) Myocardial Ischemia after Coronary Bypass: Comparison of Trimetazidine and Diltiazem. Asian Cardiovascular and Thoracic Annals, 7, 84-89. https://doi.org/10.1177/021849239900700203

[44] Ruzyllo, W., Szwed, H., Sadowski, Z., et al. (2004) Efficacy of Trimetazidine in Patients with Recurrent Angina: A Subgroup Analysis of the TRIMPOL II Study. Current Medical Research and Opinion, 20, 1447-1454. https://doi.org/10.1185/030079904X2637

[45] Banach, M., Rysz, J., Zastonka, J., et al. (2005) The Cardioprotective Effect of Trimetazidine in Patients Undergoing Surgical Revascularization. Russian Journal of Medicine, 13, 1269-1271.

[46] Iskesen, I., Saribulbul, O., Cerrahoglu, M., et al. (2006) Trimetazidine Reduces Oxidative Stress in Cardíac Surgery. Circulation Journal, 70, 1169-1173. https://doi.org/10.1253/circj.70.1169

[47] Lopatin, I.U.M. and Dronova, E.P. (2009) Clinical-Pharmacoeconomic Aspects of Trimetazidine Modified Release Use in Patients with Ischemic Heart Disease Undergoing CABG. Kardiologiia, 49, 15-21. 
[48] Martins, G.F., Siqueira, F.A.G., Santos, J.B., et al. (2012) Trimetazidine and Inflammatory Response in Coronary Artery Bypass Grafting. Arquivos Brasileiros de Cardiologia, 99, 688-696. https://doi.org/10.1590/S0066-782X2012005000066

[49] Martins, G.F., Siqueira, F.A.G., Santos, J.B., et al. (2011) Trimetazidine and Cellular Response in Cardiopulmonary Bypass. World Journal of Cardiovascular Surgery, 3, 171-179. https://doi.org/10.4236/wjcs.2013.35035

[50] Lei, H., et al. (2015) The Effectiveness of Preoperative Trimetazidine on Myocardial Preservation in Coronary Artery Bypass Graft Patients: A Systematic Review and Meta-Analysis. Cardiology, 131, 86-96. https://doi.org/10.1159/000375289

[51] Danchin, N., et al. (2011) Efficacy Comparison of Trimetazidine with Therapeutic Alternatives in Stable Angina Pectoris; a Network Meta-Analysis. Cardiology, 120, 59-72. https://doi.org/10.1159/000332369

[52] Marzilli, M. and Klein, W.W. (2003) Efficacy and Tolerability of Trimetazidinein Stable Angina: A Meta-Analysis of Randomized, Double-Blind, Controlled Trials. Coronary Artery Disease, 14, 171-179.

https://doi.org/10.1097/00019501-200304000-00010

[53] Ciapponi, A., Pizzarro, R. and Harrison, J. (2005) Trimetazidine for Stable Angina. Cochrane Database of Systematic Reviews, 4, Article ID: CD003614.

https://doi.org/10.1002/14651858.cd003614.pub2 


\section{Abbreviation List}

Adenosine 5-triphosphate (ATP)

Adenosine diphosphate (ADP)

Adenosine monophosphate (AMP)

Calcium $\left(\mathrm{Ca}^{2+}\right)$

Coronary artery bypass grafting (CABG)

Creatine kinase isoenzyme MB (CK-MB)

Creatine phosphokinase (CPK)

Deoxyribonucleic acid (DNA)

Dihydropyridine receptors (DHP)

Ejection fraction (EF)

Excitation-contraction (EC)

Fractional area change (FAC)

Glutadione peroxidase (gpx)

Highly sensitive C-reactive protein (HS-CRP)

Hydrogene peroxide $\left(\mathrm{H}_{2} \mathrm{O}_{2}\right)$

Hydroxyl radical $(\mathrm{OH})$

Intensive care unit (ICU)

Interleukin (IL)

Left ventricular (LV)

L-type calcium channels (LTCC)

Malondialdehyde (MDA)

Malondialdehyde (MDA)

Mitochondrial permeability transition pore (MPTP)

New York Heart Association (NYHA)

Nicotinamide adenine dinucleotide (NADH)

Percutaneous transluminal coronary angioplasty (PTCA)

Placebo (PCB)

Randomized controlled trials (RCTS)

Reactive oxygen species (ROS)

Ryanodine receptor type 2 (ryr2)

Sarcoplasmicreticulum (SR)

Sodiumions $\left(\mathrm{Na}^{+}\right)$

Superoxideanion $\left(\mathrm{O}_{2}^{-}\right)$

Superoxidedismutase (SOD)

Systolic wall thickening (SWT)

Tissue necrosis factor-alpha (TNF- $\alpha$ )

Trimetazidine (TMZ)

Troponin I (TNI)

Troponin $\mathrm{t}$ (TNT)

Work stroke index (SWI)

Xanthine dehydrogenase (XD)

Xanthine oxidase (XO) 
Submit or recommend next manuscript to SCIRP and we will provide best service for you:

Accepting pre-submission inquiries through Email, Facebook, LinkedIn, Twitter, etc. A wide selection of journals (inclusive of 9 subjects, more than 200 journals)

Providing 24-hour high-quality service

User-friendly online submission system

Fair and swift peer-review system

Efficient typesetting and proofreading procedure

Display of the result of downloads and visits, as well as the number of cited articles Maximum dissemination of your research work

Submit your manuscript at: http://papersubmission.scirp.org/

Or contact wjcs@scirp.org 